\title{
Clinical and microbiological characterization of carbapenem-resistant Acinetobacter baumannii bloodstream infections
}

Correspondence

Hee Jin Cheong

heejinmd@medimail.co.kr

Received 22 December 2010

Accepted 7 January 2011

\author{
Joon Young Song, Hee Jin Cheong, Won Suk Choi, Jung Yeon Heo, \\ Ji Yun Noh and Woo Joo Kim
}

\author{
Division of Infectious Disease, Department of Internal Medicine, Korea University College of \\ Medicine, Seoul, Republic of Korea
}

\begin{abstract}
The incidence of carbapenem-resistant Acinetobacter baumannii infection is increasing, which might be associated with high morbidity and mortality among critically ill patients with limited therapeutic options. This study was conducted to evaluate the clinical and microbiological features of carbapenem-resistant $A$. baumannii bacteraemia. The medical records of 28 adult patients with this bacteraemia admitted to Korea University Guro Hospital, from January 2005 through December 2010, were reviewed. Using the 28 bloodstream isolates, we intended to detect genes encoding carbapenemases, and investigate the inoculum effect on each of the antimicrobial agents rifampicin, imipenem, colistin and tigecycline. With one blood isolate from a patient with pneumonia, rifampicin-inducible resistance was examined using the experimental mouse pneumonia model. Out of 28 carbapenem-resistant $A$. baumannii bloodstream infections (Bls), the most common primary focus was the central venous catheter (35.7\%) and then the lung (32.1\%). The 30 day overall mortality was $53.6 \%$; in most cases $(80 \%)$ the patients died within 10 days after the onset of the bacteraemia. By univariate analysis, inappropriate antimicrobial therapy (73.3 vs $30.8 \%, P=0.02$ ), mechanical ventilation (53.3 vs $15.4 \%, P=0.04$ ) and a high Pitt bacteraemia score $(4.9 \pm 1.9$ vs $2.2 \pm 1.2, P<0.01)$ were statistically significant risk factors for mortality, while only a high Pitt bacteraemia score (odds ratio 2.6; $95 \%$ confidence interval 1.1-6.5) was independently associated with 30 day mortality by multivariate analysis. All 28 isolates had the bla OXA-51-like gene with upstream ISAbal, 2 of which additionally had the bla ${ }_{\mathrm{OXA}-58^{-}}$like gene and the bla $\mathrm{OXA}_{23}$-like gene. Inoculum effect and rifampicin inducible resistance were not detected. Considering the rapid progression to death in carbapenemresistant $A$. baumannii Bls, early empirical antibiotic therapy would be warranted based on the local microbiological data in each hospital.
\end{abstract}

\section{INTRODUCTION}

Acinetobacter baumannii can cause suppurative infections in virtually every organ system of the human body including pneumonia, surgical site infections, skin and soft tissue infections, urinary tract infections, postoperative meningitis and catheter-related infections (Munoz-Price \& Weinstein, 2008; Peleg et al., 2008). Bacteraemia is often associated with severe A. baumannii infections, and the overall mortality is approximately $25 \%$, and up to $54 \%$ in intensive care units (ICUs) (Bergogne-Bérézin \& Towner, 1996; Poutanen et al., 1997). Carbapenems are the preferred therapeutic option for A. baumannii infections. In the past decade, carbapenem-resistant $A$. baumannii has emerged as a significant

Abbreviations: $\mathrm{Bl}$, bloodstream infection; $\mathrm{Cl}$, confidence interval; ICU, intensive care unit. nosocomial pathogen worldwide; the carbapenem-resistance rate among A. baumannii has markedly increased to up to $60 \%$ in Korea (Lee et al., 2008). As for carbapenemresistant A. baumannii infections, colistin and tigecycline have been suggested as possible effective therapeutic choices (Maragakis \& Perl, 2008). Though rifampicinbased regimens also have shown favourable outcomes, rifampicin-induced resistance has become a concern (Saballs et al., 2006; Song et al., 2008). With limited therapeutic options, infections caused by carbapenemresistant organisms might result in greater mortality, longer hospitalization and higher costs than those caused by susceptible organisms. The clinical and microbiological data for carbapenem-resistant A. baumannii bacteraemia are not sufficient, and the impact of early appropriate antimicrobial therapy on survival continues to be debated (Wareham et al., 2008; Erbay et al., 2009). 
The goal of this study was to evaluate the clinical and microbiological features of bloodstream infections (BIs) caused by carbapenem-resistant $A$. baumannii. To better characterize the microbiological aspects of carbapenemresistant A. baumannii blood isolates, the prevalence of each type of carbapenemase, the MIC/inoculum effects of each antibiotic agent and inducible rifampicin resistance were assessed using clinical A. baumannii isolates from blood.

\section{METHODS}

Study subjects and data collection. The medical records of 28 adult patients ( $\geqslant 18$ years old) with clinically significant carbapenemresistant $A$. baumannii bacteraemia who were admitted to Korea University Guro Hospital (a 1000 bed tertiary care university hospital), from January 2005 through December 2010, were reviewed. The data collected included: age, gender, underlying illness, primary site of infection, severity of illness (as calculated by the Pitt bacteraemia score and Charlson's weighted index of morbidity), laboratory findings, antibiotic regimen, duration of hospitalization/ ICU stay and 30 day mortality. The patients were divided into two groups according to the 30 day survival after the diagnosis of bacteraemia. The following medical conditions were also documented: treatment with immunosuppressive medications within 30 days before the bacteraemia, nasogastric tube, mechanical ventilation, haemodialysis and the presence of a central venous catheter. Immunosuppressant use was defined as treatment with prednisolone ( $\geqslant 10 \mathrm{mg}$ daily) or any other immunosuppressant during the 30 days prior to the diagnosis of bacteraemia.

Clinically significant A. baumannii bacteraemia was defined as one or more positive blood cultures, together with clinical features compatible with systemic inflammatory response syndrome. When a patient had more than one bacteraemic episode, only the first episode was included. Appropriate antibiotic therapy was defined as one of the following regimens given within $48 \mathrm{~h}$ after the blood culture was obtained: carbapenem-rifampicin-, carbapenem-sulbactam-, tigecycline- and colistin-based regimens.

Characterization of $\boldsymbol{\beta}$-lactamases. In order to analyse the production of class B and D carbapenemase, carbapenem-resistant isolates were first screened by a modified Hodge test and an imipenem-EDTA double disc synergy test (Lee et al., 2001). PCR was performed to detect $b l a_{\mathrm{OXA}}, b l a_{\mathrm{IMP}}$ and $b l a_{\mathrm{VIM}}$ genes using specific primers as previously described (Song et al., 2007). The presence of the insertion element ISAbal upstream of $b l a_{\text {OXA-51-like was also }}$ investigated by PCR (Turton et al., 2006).

Antimicrobial susceptibility testing and inoculum effect. Antimicrobial susceptibilities were determined for imipenem, colistin sulfate, tigecycline and rifampicin according to Clinical and Laboratory Standard Institute guidelines using a broth microdilution method (CLSI, 2006). The bacteria were tested by two inocula sizes $\left(10^{5}\right.$ and $10^{7}$ c.f.u. $\left.\mathrm{ml}^{-1}\right)$, and were incubated in ambient air at $37^{\circ} \mathrm{C}$ for $24 \mathrm{~h}$. The lowest drug concentration showing no growth was accepted as the MIC. Each isolate was considered resistant to carbapenem if the MIC against imipenem was $\geqslant 16 \mathrm{mg}^{-1}$. The following concentrations were considered as the susceptibility breakpoints of the other tested antimicrobials: colistin, $4 \mathrm{mg}^{-1}$; tigecycline, $2 \mathrm{mg} \mathrm{l}^{-1}$; rifampicin, $2 \mathrm{mg} \mathrm{l}^{-1}$ (Giamarellos-Bourboulis et al., 2001; Pankey, 2005). The changes of the MICs according to the inoculum size for imipenem, rifampicin and colistin were compared. An inoculum effect was defined as a fourfold or greater increase in the
MIC on testing with the higher inoculum compared to the lower inoculum.

Inducible rifampicin resistance. Immunocompetent specificpathogen-free CD-1 (ICR) young female mice, weighing on average $25 \mathrm{~g}$ (6-7 weeks old), were supplied by Orient Bio. All animal procedures were performed in accordance with the guidelines of Korea University Guro Hospital for the humane handling, care and treatment of research animals. An experimental mouse pneumonia model was used to evaluate the selection of rifampicin-resistant mutants of A. baumannii (Song et al., 2009). Twenty mice were inoculated with an $A$. baumannii isolate from a patient with pneumonia and bacteraemia. Five mice were allocated to each time point $(24,48,72$ and $96 \mathrm{~h}$ after inoculation): two mice out of the five were controls (without treatment), and the other three were treated with $100 \mathrm{mg}$ rifampicin $\mathrm{kg}^{-1}$ per day. The lungs of the mice were removed at each time point and homogenized for $2 \mathrm{~min}$ in $2 \mathrm{ml}$ sterile saline. After 10-fold dilution, $0.1 \mathrm{ml}$ was plated on sheep blood agar for $24 \mathrm{~h}$ at $37{ }^{\circ} \mathrm{C}$ and the counts were expressed as $\log _{10}$ c.f.u. (g tissue $)^{-1}$. The MIC of rifampicin was determined at each time point.

Statistical analysis. Data were analysed with SPSS 10.0 (SPSS). A $P$ value of $<0.05$ was considered statistically significant. Student's $t$-test was used to compare continuous variables, and the Fisher exact test was used to compare categorical variables. Multivariate analysis was carried out using a stepwise logistic regression model.

\section{RESULTS}

\section{Clinical characteristics and risk factors for mortality among patients with Acinetobacter bacteraemia}

All 28 cases of Acinetobacter BI were nosocomial, and $89.3 \%$ (25 among 28 ) of cases developed during an ICU stay. The median age of patients was 64 (range 23-88 years) and half of the patients were men (Table 1). The most common primary site of infection was a central venous catheter (10 patients; $35.7 \%$ ), followed by the lung ( 9 patients; $32.1 \%$ ), surgical site wound (3 patients; $10.7 \%$ ), urinary tract (3 patients; $10.7 \%)$, intra-abdominal focus $(2$ patients; $7.1 \%$ ) and unknown sites (1 patient; $3.6 \%$ ). The finding of preceding colonization was identified in only five patients $(17.9 \%)$. A total of 3 out of 28 patients $(10.7 \%)$ presented with polymicrobial bacteraemia.

The 30 day overall mortality was $53.6 \%$. In 12 of the 15 fatal cases $(80 \%)$ the patients passed away within 10 days after the onset of the bacteraemia; the median interval from bacteraemia onset to death was 7 days (range 3-30 days). When the characteristics of those who survived were compared to the patients who did not survive, the demographic and clinical characteristics were indistinguishable for age, gender, underlying medical conditions, primary site of infection, Charlson's weighted index of co-morbidity, immunosuppressant use and hospital/ICU stay before onset of bacteraemia (Table 1). Inappropriate antimicrobial therapy $(73.3$ vs $30.8 \%, P=0.02)$, mechanical ventilation (53.3 vs $15.4 \%, P=0.04)$ and a high Pitt bacteraemia score $(4.9 \pm 1.9$ vs $2.2 \pm 1.2, P<0.01)$ were statistically significant risk factors of mortality. For multivariate analysis, only a 
Table 1. Demographic and clinical characteristics of patients with carbapenem-resistant $A$. baumannii $\mathrm{BI}$ : comparison based on the clinical outcome

\begin{tabular}{|c|c|c|c|c|}
\hline Patient characteristic & Survived $(n=13)$ & Died $(n=15)$ & Total $(n=28)$ & $P$ value \\
\hline Age (years) & $57.7 \pm 18.9$ & $62.5 \pm 15.1$ & $60.3 \pm 16.8$ & 0.46 \\
\hline Sex: no. of males (\%) & $6(46.2)$ & $9(60.0)$ & $15(53.6)$ & 0.46 \\
\hline Diabetes mellitus & $5(38.5)$ & $3(20.0)$ & $8(28.6)$ & 0.28 \\
\hline Chronic liver diseases & $3(23.1)$ & $3(20.0)$ & $6(21.4)$ & 0.84 \\
\hline Chronic renal insufficiency & $2(15.4)$ & $4(26.7)$ & $6(21.4)$ & 0.47 \\
\hline Haematological malignancy & $0(0)$ & $3(20.0)$ & $3(10.7)$ & 0.09 \\
\hline Solid tumour & $2(15.4)$ & $1(6.7)$ & $3(10.7)$ & 0.46 \\
\hline Chronic obstructive lung diseases & $1(7.7)$ & $1(6.7)$ & $2(7.1)$ & 0.27 \\
\hline Congestive heart failure & $1(7.7)$ & $1(6.7)$ & $2(7.1)$ & 0.92 \\
\hline Charlson's weighted index of co-morbidity & $4.0 \pm 1.7$ & $4.9 \pm 2.7$ & $4.5 \pm 2.3$ & 0.29 \\
\hline Pitt bacteraemia score & $2.2 \pm 1.2$ & $4.9 \pm 1.9$ & $3.6 \pm 2.1$ & $<0.01$ \\
\hline Intra-abdominal focus & $2(15.4)$ & $0(0)$ & $2(7.1)$ & - \\
\hline Surgical site wound & $0(0)$ & $3(20.0)$ & $3(10.7)$ & - \\
\hline Unknown origin & $0(0)$ & $1(6.7)$ & $1(3.6)$ & - \\
\hline Polymicrobial bacteraemia & $2(15.4)$ & $1(6.7)$ & $3(10.7)$ & 0.46 \\
\hline \multicolumn{5}{|l|}{ Risk factors } \\
\hline Immunosuppressant use & $2(15.4)$ & $7(46.7)$ & $9(32.1)$ & 0.08 \\
\hline Nasogastric tube & $8(61.5)$ & $11(73.3)$ & $19(67.9)$ & 0.51 \\
\hline Total parenteral nutrition & $5(38.5)$ & $9(60.0)$ & $14(50.0)$ & 0.26 \\
\hline Mechanical ventilation & $2(15.4)$ & $8(53.3)$ & $10(35.7)$ & 0.04 \\
\hline Haemodialysis & $2(15.4)$ & $1(6.7)$ & $3(10.7)$ & 0.46 \\
\hline Hospital stay before bacteraemia (days) & $23.9 \pm 25.5$ & $31.7 \pm 34.5$ & $28.1 \pm 30.4$ & 0.50 \\
\hline
\end{tabular}

high Pitt bacteraemia score [odds ratio 2.6; $95 \%$ confidence interval (CI) 1.1-6.5] was independently associated with 30 day mortality; the odds ratios for mortality were 2.1 (95\% CI 0.2-23.3) with inappropriate antimicrobial therapy, and 5.2 (95\% CI 0.4-61.9) with mechanical ventilation. In the cases with catheter-related infections, catheter removal was the most important factor for patient survival; none of the infected patients survived without catheter removal $(83.3 \%$ with removal vs $0 \%$ without removal, $P=0.02$ ) (Table 2 ). Meropenem-rifampicin was effective against pneumonia with low level carbapenemresistant $A$. baumannii regardless of the rifampicin MIC; $72.7 \%$ (8 among 11 cases) survived with meropenemrifampicin treatment. Three out of four fatal cases with rifampicin-based regimens were related to high-level rifampicin resistance $\left(\mathrm{MIC}>128 \mathrm{mg} \mathrm{l}^{-1}\right.$ ) (Table 2$)$.

\section{Antimicrobial susceptibility testing and inoculum effect}

In the total of 28 tested A. baumannii isolates, rifampicin, imipenem, colistin and tigecycline were not associated with an inoculum effect. The $\mathrm{MIC}_{50 \text { s }}$ of rifampicin, imipenem, colistin, and tigecycline were $4,32,4$ and $2 \mathrm{mg} \mathrm{l}^{-1}$, respectively, with the standard inoculum tests, which was not increased more than twofold with the higher inoculum tests (Table 2). Four A. baumannii isolates had a rifampicin MIC $>128 \mathrm{mg} \mathrm{l}^{-1}$ regardless of the inoculum size.

\section{Characterization of $\boldsymbol{\beta}$-lactamases}

All carbapenem-resistant A. baumannii isolates were positive by the modified Hodge test, but negative by the imipenem-EDTA double disc synergy test. All 28 isolates carried the $b l a_{\mathrm{OXA}-51}$-like gene, 2 of which also had the

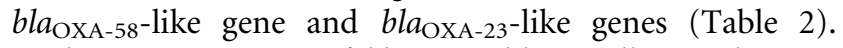

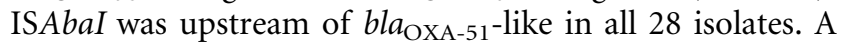
metallo- $\beta$-lactamase producing organism was not detected.

\section{Induced rifampicin resistance}

The MICs of rifampicin did not increase with rifampicin treatment (600 mg per day). They were maintained around $8 \mathrm{mg} \mathrm{l}^{-1}$ for up to $96 \mathrm{~h}$ in both the treatment and control groups (Table 3 ). 
Table 2. Characterization of carbapenemase and standard/high inoculum MICs of 20 carbapenem-resistant $A$. baumannii isolates

\begin{tabular}{|c|c|c|c|c|c|c|c|c|c|c|c|}
\hline \multirow[t]{3}{*}{$\begin{array}{l}\text { Primary infection site } \\
\text { for each patient }\end{array}$} & \multirow[t]{3}{*}{$\begin{array}{c}30 \text { day } \\
\text { mortality }\end{array}$} & \multirow[t]{3}{*}{$\begin{array}{l}\text { Appropriateness of } \\
\text { antibiotic regimen }\end{array}$} & \multirow{3}{*}{$\begin{array}{l}\text { Type of } \\
\text { carbape- } \\
\text { nemase }\end{array}$} & \multicolumn{8}{|c|}{$\begin{array}{c}\text { MIC }\left(\mathrm{mg} \mathrm{l}^{-1}\right) \text { of each agent at inocula of } 10^{5} \\
\text { and } 10^{7} \text { c.f.u. } \mathrm{ml}^{-1}\end{array}$} \\
\hline & & & & \multicolumn{2}{|c|}{ Rifampicin ${ }^{\star}$} & \multicolumn{2}{|c|}{ Imipenem $\dagger$} & \multicolumn{2}{|c|}{ Colistin $\ddagger$} & \multicolumn{2}{|c|}{ Tigecycline $\S$} \\
\hline & & & & $10^{5}$ & $10^{7}$ & $10^{5}$ & $10^{7}$ & $10^{5}$ & $10^{7}$ & $10^{5}$ & $10^{7}$ \\
\hline Pneumonia & Dead & Meropenem (I) & OXA-51 & 4 & 8 & 16 & 32 & 4 & 8 & 2 & 4 \\
\hline Catheter-related infectionll & Survived & Meropenem (I) & OXA-51 & 4 & 8 & 64 & 128 & 4 & 8 & 2 & 4 \\
\hline Surgical site infection & Dead & Ciprofloxacin (I) & OXA-51 & 4 & 4 & 32 & 128 & 4 & 4 & 2 & 4 \\
\hline Catheter-related infectionll & Dead & Colistin (I) & OXA-51 & 128 & 128 & 64 & 128 & 8 & 8 & 2 & 2 \\
\hline Catheter-related infectionll & Survived & Colistin (A) & OXA-51 & $>128$ & $>128$ & $>128$ & $>128$ & 4 & 8 & 1 & 2 \\
\hline Unknown & Dead & None (I) & OXA-51 & 4 & 8 & 32 & 64 & 2 & 4 & 2 & 2 \\
\hline Pneumonia & Survived & $\begin{array}{l}\text { Meropenem + rifampicin } \\
\text { (I) }\end{array}$ & OXA-51 & 4 & 8 & 16 & 16 & 4 & 8 & 2 & 2 \\
\hline Catheter-related infection & Dead & Colistin + rifampicin (I) & OXA-51 & $>128$ & $>128$ & 16 & 32 & 4 & 8 & 2 & 2 \\
\hline Catheter-related infection & Dead & $\begin{array}{l}\text { Meropenem + rifampicin } \\
\text { (A) }\end{array}$ & OXA-51 & 4 & 8 & 64 & 128 & 4 & 8 & 1 & 2 \\
\hline Catheter-related infectionll & Survived & Colistin + meropenem (A) & OXA-51 & 8 & 8 & 16 & 16 & 4 & 8 & 1 & 2 \\
\hline Catheter-related infection & Dead & Meropenem (I) & OXA-51 & 8 & 8 & 16 & 32 & 4 & 8 & 1 & 2 \\
\hline Pneumonia & Dead & $\begin{array}{l}\text { Meropenem + rifampicin } \\
\text { (I) }\end{array}$ & OXA-51 & $>128$ & $>128$ & 64 & 128 & 4 & 8 & 1 & 2 \\
\hline Intra-abdominal infection & Survived & Colistin + meropenem (A) & OXA-51 & 8 & 8 & 16 & 32 & 4 & 8 & 1 & 2 \\
\hline Pneumonia & Survived & $\begin{array}{l}\text { Meropenem + rifampicin } \\
\text { (A) }\end{array}$ & $\begin{array}{l}\text { OXA-51, } \\
\text { OXA-58 }\end{array}$ & 4 & 8 & 32 & 32 & 4 & 16 & 0.25 & 1 \\
\hline Urinary tract infection & Survived & Colistin (A) & OXA-51 & 4 & 4 & 64 & 128 & 2 & 2 & 1 & 2 \\
\hline Pneumonia & Dead & Tigecycline (A) & OXA-51 & 4 & 8 & 64 & 128 & 2 & 2 & 1 & 2 \\
\hline Pneumonia & Dead & None (I) & OXA-51 & 4 & 8 & 64 & 64 & 2 & 4 & 2 & 4 \\
\hline $\begin{array}{l}\text { Catheter-related } \\
\text { infectionll }\end{array}$ & Survived & $\begin{array}{l}\text { Meropenem + rifampicin } \\
\text { (A) }\end{array}$ & OXA-51 & 4 & 4 & 32 & 32 & 2 & 2 & 2 & 4 \\
\hline Urinary tract infection & Dead & None (I) & OXA-51 & 4 & 8 & 32 & 64 & 2 & 4 & 1 & 2 \\
\hline Pneumonia & Survived & $\begin{array}{l}\text { Meropenem + rifampicin } \\
\text { (A) }\end{array}$ & OXA-51 & 4 & 4 & 16 & 32 & 2 & 2 & 1 & 2 \\
\hline Urinary tract infection & Survived & Meropenem (I) & OXA-51 & 4 & 4 & 32 & 32 & 1 & 2 & 0.5 & 1 \\
\hline $\mathrm{MIC}_{50}$ & & & & 4 & 8 & 32 & 64 & 4 & 8 & 2 & 2 \\
\hline $\mathrm{MIC}_{90}$ & & & & $>128$ & $>128$ & 64 & 128 & 4 & 8 & 2 & 4 \\
\hline
\end{tabular}

A, Appropriate; I, inappropriate.

${ }^{\star}$ Susceptible $\leqslant 2 \mathrm{mg} \mathrm{ml}^{-1}$, resistant $\geqslant 4 \mathrm{mg} \mathrm{ml}^{-1}$; working party report of the British Society for Antimicrobial Chemotherapy.

$\dagger$ Susceptible $\leqslant 4 \mathrm{mg} \mathrm{ml}^{-1}$, resistant $\geqslant 16 \mathrm{mg} \mathrm{ml}^{-1}$; Clinical and Laboratory Standards Institute.

$\ddagger$ Susceptible $\leqslant 4 \mathrm{mg} \mathrm{ml}^{-1}$, resistant $\geqslant 8 \mathrm{mg} \mathrm{ml}^{-1}$; British Society for Antimicrobial Chemotherapy.

$\S$ Susceptible $\leqslant 2 \mathrm{mg} \mathrm{ml}^{-1}$, resistant $\geqslant 8 \mathrm{mg} \mathrm{ml}^{-1}$ by Wyeth Research.

IICatheter was removed. 
Table 3. Rifampicin MICs for carbapenem-resistant $A$. baumannii isolates 24, 48, 72 and $96 \mathrm{~h}$ after treatment with rifampicin

\begin{tabular}{|c|c|c|c|}
\hline $\begin{array}{l}\text { Time interval after } \\
\text { rifampicin trial (h) }\end{array}$ & Mouse no. & Cell count $\left[\log\right.$ c.f.u. $\left.(\mathrm{g} \text { tissue })^{-1}\right]$ & $\operatorname{MIC}\left(\mathrm{mg} \mathrm{l}^{-1}\right)$ \\
\hline \multirow[t]{4}{*}{24} & 1 & 4.96 & 8 \\
\hline & 3 & 4.38 & 8 \\
\hline & Control 1 & 9.58 & 8 \\
\hline & Control 2 & 9.28 & 8 \\
\hline \multirow{4}{*}{48} & 2 & 1.54 & 8 \\
\hline & 3 & 2.39 & 8 \\
\hline & Control 1 & 9.70 & 8 \\
\hline & Control 2 & 9.23 & 8 \\
\hline \multirow[t]{2}{*}{72} & 1 & 1.64 & 4 \\
\hline & 2 & 1.48 & 8 \\
\hline \multirow{3}{*}{96} & 2 & 2.42 & 8 \\
\hline & 3 & 1.34 & 8 \\
\hline & Control & No survivors & \\
\hline
\end{tabular}

\section{DISCUSSION}

In this study, the clinical and microbiological characteristics of bacteraemia due to carbapenem-resistant $A$. baumannii are described. Clinical manifestations of $A$. baumannii induced BIs ranged from self limiting, transient bacteraemia to fulminant disease with a high mortality (Seifert et al., 1995). The overall mortality rate of Acinetobacter bacteraemia has been reported to be high, ranging from 22 to $61.6 \%$ according to the study population (Bergogne-Bérézin \& Towner, 1996; Kwon et al., 2007; Wareham et al., 2008; Erbay et al., 2009; Metan et al., 2009). In this study, the overall mortality was $53.6 \%$, with rates of 30.8 and $73.3 \%$ for patients who received appropriate and inappropriate antibiotic therapy within $48 \mathrm{~h}$, respectively. Compared to previous studies, the mortality rate was remarkably high, and in most cases the patients died within 10 days after the onset of the bacteraemia. The differences in this study might have been due to the severity of illness as shown by the high Pitt bacteraemia score; only carbapenem-resistant cases were included. Because routine surveillance cultures were not performed for A. baumannii, preceding colonization was documented in only five patients $(17.9 \%)$. In the endemic setting of carbapenem-resistant A. baumannii, empirically targeted antibiotic treatment and late de-escalation of treatment would be warranted. Consistent with previous reports, most cases had the risk factors for A. baumannii infection including a central venous catheter, total parenteral nutrition, a nasogastric tube and mechanical ventilation; catheter-related infections and pneumonias were the most common primary focus of bacteraemia (Glew et al., 1977). In cases with catheter-related BI, catheter removal was invariably required.
Carbapenem-resistant A. baumannii infection has been increasing and this is a significant concern. A recent study in Korea reported that most isolates acquired low-level carbapenem resistance due to the upregulation of the OXA-51-like enzyme; enzyme expression is known to be variable according to the presence of ISAba (Héritier et al., 2006; Poirel \& Nordmann, 2006; Corvec et al., 2007). Likewise, ISAbaI was upstream of bla $a_{\text {OXA-51-like in all } 28}$ isolates in this study. Meropenem-rifampicin- and colistin-based combinations have been regarded as effective regimens, and use of the appropriate antibiotic therapy was related to the clinical outcome in this study.

It is assumed that high bacterial loads in patients with severe infection would affect the antibiotic response. Among carbapenem-resistant A. baumannii isolates, however, an inoculum effect was not found with any of the antibiotic agents imipenem, colistin, rifampicin and tigecycline. Rifampicin, considered inactive against Gramnegative bacteria, has emerged as one of the therapeutic options for infections caused by carbapenem-resistant $A$. baumannii. It is hypothesized that substantial changes in the outer membrane of multi-drug resistant $A$. baumannii may enable greater access to the target site (Li et al., 2007). When it comes to combination with other antibiotics, colistin is known to induce damage to the cell-wall structures of Gram-negative bacteria, thereby allowing the accelerated penetration of rifampicin (Song et al., 2008; Aoki et al., 2009). Moreover, Bernabeu-Wittel et al. (2004) reported that meropenem at sub-MIC levels induced spheroplastic changes $(>3 \mu \mathrm{m}$ in size) of A. baumannii isolates, which might also increase the intracellular penetration of rifampicin. 
Although some previous studies reported inducible rifampicin resistance during treatment (Pachón-Ibáñez et al., 2006; Saballs et al., 2006), a change of the rifampicin MIC was not found up to $96 \mathrm{~h}$ after treatment in this study. However, high-level resistance (MIC $>128 \mathrm{mg} \mathrm{l}^{-1}$ ) would be predictive of a poor clinical and microbiological response as previously reported (Song et al., 2008).

In conclusion, low-level carbapenem-resistant A. baumannii strains with OXA-51-like carbapenemase were prevalent in the study population. Considering the rapid progression to death in carbapenem-resistant A. baumannii BIs, early empirical antibiotic therapy would be warranted based on the local microbiological data in each hospital.

\section{ACKNOWLEDGEMENTS}

This work was supported by a Korea University grant.

\section{REFERENCES}

Aoki, N., Tateda, K., Kikuchi, Y., Kimura, S., Miyazaki, C., Ishii, Y., Tanabe, Y., Gejyo, F. \& Yamaguchi, K. (2009). Efficacy of colistin combination therapy in a mouse model of pneumonia caused by multidrug-resistant Pseudomonas aeruginosa. J Antimicrob Chemother 63, 534-542.

Bergogne-Bérézin, E. \& Towner, K. J. (1996). Acinetobacter spp. as nosocomial pathogens: microbiological, clinical, and epidemiological features. Clin Microbiol Rev 9, 148-165.

Bernabeu-Wittel, M., García-Curiel, A., Pichardo, C., Pachón-Ibáñez, M. E., Jiménez-Mejías, M. E. \& Pachón, J. (2004). Morphological changes induced by imipenem and meropenem at sub-inhibitory concentrations in Acinetobacter baumannii. Clin Microbiol Infect 10, 931-934.

Corvec, S., Poirel, L., Naas, T., Drugeon, H. \& Nordmann, P. (2007). Genetics and expression of the carbapenem-hydrolyzing oxacillinase gene blaOXA-23 in Acinetobacter baumannii. Antimicrob Agents Chemother 51, 1530-1533.

Erbay, A., Idil, A., Gözel, M. G., Mumcuoğlu, I. \& Balaban, N. (2009). Impact of early appropriate antimicrobial therapy on survival in Acinetobacter baumannii bloodstream infections. Int J Antimicrob Agents 34, 575-579.

Giamarellos-Bourboulis, E. J., Xirouchaki, E. \& Giamarellou, H. (2001). Interactions of colistin and rifampin on multidrugresistant Acinetobacter baumannii. Diagn Microbiol Infect Dis 40, 117-120.

Glew, R. H., Moellering, R. C., Jr \& Kunz, L. J. (1977). Infections with Acinetobacter calcoaceticus (Herellea vaginicola): clinical and laboratory studies. Medicine (Baltimore) 56, 79-97.

Héritier, C., Poirel, L. \& Nordmann, P. (2006). Cephalosporinase overexpression resulting from insertion of ISAba1 in Acinetobacter baumannii. Clin Microbiol Infect 12, 123-130.

Kwon, K. T., Oh, W. S., Song, J. H., Chang, H. H., Jung, S. I., Kim, S. W., Ryu, S. Y., Heo, S. T., Jung, D. S. \& other authors (2007). Impact of imipenem resistance on mortality in patients with Acinetobacter bacteraemia. J Antimicrob Chemother 59, 525-530.

Lee, K., Chong, Y., Shin, H. B., Kim, Y. A., Yong, D. \& Yum, J. H. (2001). Modified Hodge and EDTA-disk synergy tests to screen metallo- $\beta$-lactamase-producing strains of Pseudomonas and Acinetobacter species. Clin Microbiol Infect 7, 88-91.

Lee, S. O., Kim, E. S., Kim, H. Y., Park, E. S., Jin, H. Y., Ki, H. K., Choi, H. J., Jeong, S. Y., Kim, S. R. \& other authors (2008). [Korean nosocomial infections surveillance system, intensive care unit module report: data summary from July 2007 through June 2008]. Korean J Nosocomial Infect Control 13, 69-82 (in Korean).

Li, J., Nation, R. L., Owen, R. J., Wong, S., Spelman, D. \& Franklin, C. (2007). Antibiograms of multidrug-resistant clinical Acinetobacter baumannii: promising therapeutic options for treatment of infection with colistin-resistant strains. Clin Infect Dis 45, 594-598.

Maragakis, L. L. \& Perl, T. M. (2008). Acinetobacter baumannii: epidemiology, antimicrobial resistance, and treatment options. Clin Infect Dis 46, 1254-1263.

Metan, G., Sariguzel, F. \& Sumerkan, B. (2009). Factors influencing survival in patients with multi-drug-resistant Acinetobacter bacteraemia. Eur J Intern Med 20, 540-544.

Munoz-Price, L. S. \& Weinstein, R. A. (2008). Acinetobacter infection. $N$ Engl J Med 358, 1271-1281.

Pachón-Ibáñez, M. E., Fernández-Cuenca, F., Docobo-Pérez, F., Pachón, J. \& Pascual, A. (2006). Prevention of rifampicin resistance in Acinetobacter baumannii in an experimental pneumonia murine model, using rifampicin associated with imipenem or sulbactam. J Antimicrob Chemother 58, 689-692.

Pankey, G. A. (2005). Tigecycline. J Antimicrob Chemother 56, 470480.

Peleg, A. Y., Seifert, H. \& Paterson, D. L. (2008). Acinetobacter baumannii: emergence of a successful pathogen. Clin Microbiol Rev 21, 538-582.

Poirel, L. \& Nordmann, P. (2006). Genetic structures at the origin of acquisition and expression of the carbapenem-hydrolyzing oxacillinase gene bla $a_{\mathrm{OXA}-58}$ in Acinetobacter baumannii. Antimicrob Agents Chemother 50, 1442-1448.

Poutanen, S. M., Louie, M. \& Simor, A. E. (1997). Risk factors, clinical features and outcome of Acinetobacter bacteremia in adults. Eur J Clin Microbiol Infect Dis 16, 737-740.

Saballs, M., Pujol, M., Tubau, F., Peña, C., Montero, A., Domínguez, M. A., Gudiol, F. \& Ariza, J. (2006). Rifampicin/imipenem combination in the treatment of carbapenem-resistant Acinetobacter baumannii infections. J Antimicrob Chemother 58, 697-700.

Seifert, H., Strate, A. \& Pulverer, G. (1995). Nosocomial bacteremia due to Acinetobacter baumannii: clinical features, epidemiology, and predictors of mortality. Medicine (Baltimore) 74, 340-349.

Song, J. Y., Kee, S. Y., Hwang, I. S., Seo, Y. B., Jeong, H. W., Kim, W. J. \& Cheong, H. J. (2007). In vitro activities of carbapenem/sulbactam combination, colistin, colistin/rifampicin combination and tigecycline against carbapenem-resistant Acinetobacter baumannii. J Antimicrob Chemother 60, 317-322.

Song, J. Y., Lee, J., Heo, J. Y., Noh, J. Y., Kim, W. J., Cheong, H. J. \& Hwang, I. S. (2008). Colistin and rifampicin combination in the treatment of ventilator-associated pneumonia caused by carbapenemresistant Acinetobacter baumannii. Int J Antimicrob Agents 32, 281284.

Song, J. Y., Cheong, H. J., Lee, J., Sung, A. K. \& Kim, W. J. (2009). Efficacy of monotherapy and combined antibiotic therapy for carbapenem-resistant Acinetobacter baumannii pneumonia in an immunosuppressed mouse model. Int J Antimicrob Agents 33, 33-39.

Turton, J. F., Ward, M. E., Woodford, N., Kaufmann, M. E., Pike, R., Livermore, D. M. \& Pitt, T. L. (2006). The role of ISAba1 in expression of OXA carbapenemase genes in Acinetobacter baumannii. FEMS Microbiol Lett 258, 72-77. 
Wareham, D. W., Bean, D. C., Khanna, P., Hennessy, E. M., Krahe, D., Ely, A. \& Millar, M. (2008). Bloodstream infection due to Acinetobacter spp: epidemiology, risk factors and impact of multi-drug resistance. Eur J Clin Microbiol Infect Dis 27, 607-612.
CLSI (2006). Methods for Dilution Antimicrobial Susceptibility Tests for Bacteria that Grow Aerobically, approved standard, 7th edn, M7-A7. Wayne, PA: Clinical and Laboratory Standards Institute. 